\title{
Neighborhood predictors of bullying perpetration and victimization trajectories among South Korean adolescents
}

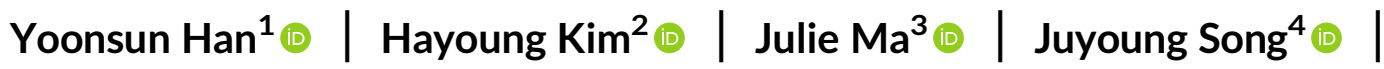 \\ Hyunhee Hong ${ }^{5}$
}

${ }^{1}$ Department of Social Welfare, Seoul National University, Gwanak-Gu, Korea

${ }^{2}$ Youth Career Development Center, National Youth Policy Institute, Sejong-si, Korea

${ }^{3}$ Department of Social Work, University of Michigan-Flint, Flint, Michigan

${ }^{4}$ Administration of Justice, Pennsylvania State University, Schuylkill Haven, Pennsylvania

${ }^{5}$ Department of Child Psychology and Education, Sungkyunkwan University, Jongno-Gu, Korea

\section{Correspondence}

Juyoung Song, PhD, Administration of Justice, Pennsylvania State University, 200 University Drive, Schuylkill Haven, PA 17972.

Email: jxs6190@psu.edu

\section{Funding information}

Ministry of Education of the Republic of Korea, National Research Foundation of Korea, Grant/Award Number:

NRF-2016S1A5A2A03925702

\author{
Abstract \\ Aims: This study examined latent trajectories of bullying \\ perpetration and victimization, and identified neighborhood \\ antecedents of these trajectories among South Korean \\ adolescents.
}

Methods: Nationally representative individual-level data from waves 2 to 6 (middle school to high school) of the Korean Children and Youth Panel Survey were merged with neighborhood-level data drawn from the Korean Census and the Korean Ministry of Education. Latent class growth analysis $(N=2,178)$ and logistic regression were conducted $(N=2,021)$. Results: Three unique trajectories of bullying experiencelow-risk (80.8\%), transient (13.3\%), high-risk (5.9\%)-were identified. Neighborhood factors (e.g., public assistance receipt, marital status, official bullying incidents, collective efficacy) predicted these distinct developmental paths.

Conclusion: Joint trajectories of perpetration and victimization can inform service or policy decisions as each developmental path may represent unique experiences for youth in need of specific resources for treatment or intervention. Neighborhood indicators are important predictors of developmental trajectories of bullying experience among adolescents.

\section{KEYWORDS}

adolescent well-being, bullying, latent class growth analysis, logistic regression, neighborhood, Republic of Korea, trajectory 


\section{1 | INTRODUCTION}

Bullying is a worldwide problem that affects all cultures and regions. Bullying is conceptualized as a form of systematic and ongoing aggression prompted by an individual or group with the purpose to obtain power, prestige, or material goods (Espelage \& Swearer, 2003). Bullying may occur in direct forms of physical aggression (e.g., hitting, pushing, kicking, and extorting) and verbal aggression (e.g., name-calling, teasing or mocking in a hurtful way, and threatening), as well as in indirect forms, such as relational aggression (e.g., social exclusion, spreading rumors; Juvonen \& Graham, 2014; Wang, lannotti, \& Nansel, 2009).

Bullying experience, both as a perpetrator or as a victim is associated with continuous adverse effects on psychological and behavioral outcomes. A meta-analytic study found that both perpetration and victimization experience in adolescence predicted aggression, delinquency, offending, and violence later in life (Ttofi, Farrington, \& Lösel, 2012). Specifically, studies have reported the associations of experience as a perpetrator with heavy drinking and marijuana use at age 21 (Kim, Catalano, Haggerty, \& Abbott, 2011), as well as greater involvement in domestic violence (Corvo, 2010). Also, bullying experience during childhood-as a bully, victim, or bully-victimplaces young adults at greater risk of psychiatric disorders (Copeland, Wolke, Angold, \& Costello, 2013).

The extent to which youth are involved in bullying perpetration and victimization change as they get older and enter higher-level schools (Espelage \& Swearer, 2003; Khoury-Kassabri, Benbenishty, Avi Astor, \& Zeira, 2004; Pellegrini \& Bartini, 2000). Developmental tasks and goals, the nature of peer relationships, and societal expectations may account for the changing patterns of bullying over time. Although an accurate identification and understanding of these differing trajectories of bullying are warranted for effective prevention and intervention, relatively few research has examined the longitudinal patterns of bullying in adolescence within a broader ecological perspective (Foster \& Brooks-Gunn, 2013). Another limitation in the bullying literature is that numerous studies have identified individual and family predictors, and to some extent school predictors, but research that examines neighborhood- or community-level factors of bullying experience remains scarce (Foster \& Brooks-Gunn, 2013; Hong \& Espelage, 2012; Low \& Espelage, 2014; Schmidt, Pierce, \& Stoddard, 2016). An understanding of the ecological factors that predict the unique developmental paths of bullying perpetration and victimization is necessary to prevent both short-term and long-term detrimental outcomes. Moreover, examining such topic in a collectivist country, such as South Korea (Korea, hereafter), may offer implications that are distinct from that of studies conducted primarily in non-collectivist Western countries. Collectivist features specific to Korea, such as public labeling of the victim within the group (Kwon, 1999), the rigid classroom structure in secondary school (Lee, Kwon, Kim, Na, \& Park, 2016), as well as the most common form of bullying being primarily group-based (Hong \& Eamon, 2009; Kwon, 1999), emphasize the significance of examining neighborhood predictors of bullying trajectories in Korea.

\section{1 | Trajectories of bullying}

Prior research has found greater temporal instability than stability in bullying involvement (Juvonen \& Graham, 2014). That is, the degree of involvement in perpetration and victimization is subject to change at different stages of development. In general, bullying perpetration and victimization peaks in early to middle adolescence, and decreases in high school (Chang, 2013; Espelage \& Swearer, 2003; Pellegrini \& Bartini, 2000). The unique developmental characteristics of early to middle adolescence may attribute for this change in bullying behavior. For example, heightened skepticism toward adult values and norms may lead youth to undermine the adverse effects of peer aggression (Pellegrini \& Long, 2002). Equally, social-cognitive bias may create a positive evaluation of bullying behavior, which enforces and normalizes the use of aggressive tactics toward peers (Crick \& Dodge, 1996). During a developmental period in which identity search and establishment of social roles are important, physical changes in puberty may also foster a vulnerable environment, as youth strive to gain higher social status in peer relationships by using bullying tactics (Yeo \& Kim, 2019). Furthermore, a fair amount of research has found that participant roles 
may change, such that victims become bullies, and bullies become victims, or youth are involved in multiple participant roles (Han, Lee, Kim, \& Lee, 2016; Kwak, Kim, \& Kim, 2016; Moon, Morash, \& McCluskey, 2012).

\subsection{Neighborhood predictors of bullying}

The ecological perspective underscores the interdependent associations between proximal and distal systems and its joint effects on human development (Bronfenbrenner, 1979). Prior literature clearly demonstrates the reciprocal influences of multiple contexts including individual, family, peer group, school, and neighborhood on bullying and victimization (Cook, Williams, Guerra, Kim, \& Sadek, 2010; Schmidt et al., 2016). Particularly, neighborhood factors may be highly influential in shaping adolescent experiences of bullying perpetration or victimization, as the school is situated within the neighborhood (Schmidt et al., 2016). Developmentally, adolescence is a transitional period during which youth strive to gain independence from their family, become more attached to their peers, and spend more time with peers in their neighborhoods with greater autonomy. Indeed, a recent meta-analysis has found significant effects of neighborhood factors (i.e., socioeconomic indicators, rates of violence or crime, and drug trafficking) on both bullying perpetration and victimization among youth (Cook et al., 2010).

Reflecting the increasing significance of neighborhood contexts during adolescence, a robust body of literature reports that multiple aspects of the neighborhood including socioeconomic and structural characteristics and social processes predict developmental outcomes of youth (for a review see Leventhal \& Brooks-Gunn, 2000). Social disorganization theory (Shaw \& McKay, 1942) offers broad explanations for the link between disadvantaged structural characteristics of neighborhoods (i.e., poverty, unemployment, single parenthood, and residential instability) and risk behavior of youth. This theory suggests that impoverished neighborhoods lack informal and formal institutions that regulate resident behavior such as youth crime and delinquency (Espelage \& Swearer, 2009).

Drawing from social disorganization theory, ideas of collective efficacy offers explanations for the link between neighborhood social processes and bullying. Collective efficacy theory posits that neighborhoods with disadvantaged conditions have low levels of trust and social cohesion, which is the ability of residents to form supportive social relationships and maintain community norms (Sampson, Raudenbush, \& Earls, 1997). Consequently, adult residents in neighborhoods with low levels of social cohesion and trust may be less inclined to collectively intervene when they observe problematic youth behavior such as violence, crime, delinquency (Odgers et al., 2009; Sampson et al., 1997), mental health problems (Xue, Leventhal, Brooks-Gunn, \& Earls, 2005), as well as bullying in their communities (Cook et al., 2010; De Frutos, 2013; Espelage \& Swearer, 2009).

\section{3 | Unique Korean context}

Bullying perpetration and victimization are serious social problems worldwide (Nansel, Craig, Overpeck, Saluja, \& Ruan, 2004); however, the broader cultural setting might influence youth behaviors that are linked to bullying. Korea provides an especially unique context because of its collectivist culture. In East Asian countries in which collectivism is highly valued (e.g., Korea), group norms are more likely to manipulate bullying involvement than it does in individualistic cultures such as Western countries (Koo, Kwak, \& Smith, 2008). Given that a common motivation for bullying perpetration is deviance of the victim from group norms and values (Teräsahjo \& Salmivalli, 2003), the tendency of majority youths ostracizing marginalized youths who violate group norms might be stronger in collectivistic cultures compared with individualistic cultures (Lee, 2006). Although not many exist, empirical studies have found that bullying perpetration and victimization in collectivist cultures are different from individualist cultures. For example, Koo et al. (2008) found that there are more perpetrators than victims in Korea, a finding that is in contrast to those based on North American samples.

Collectivist culture may play a unique role in the experiences of bullying among Korean youth. The prevalence of bullying experience in Korea-as a perpetrator, victim, or both-varies widely depending on the sample 
characteristics or definition of bullying used. According to recent estimates (Kim, 2018; Kim \& Hong, 2018; Kim, Hong, \& Jung, 2017; Ministry of Education, 2013; Park \& Oh, 2018) bullying prevalence is as low as $1.3 \%$ (Ministry of Education, 2018) to 31.7\% (Park \& Oh, 2018). In particular, the influence of collectivist culture on peer victimization in Korea may be reflected in the fact that group ostracism (17.2\%) is the second most prevalent form of victimization, followed by verbal aggression (34.7\%; Ministry of Education, 2018), which is often used as a means of isolating others.

Bullying in Korea reflects several collective characteristics that are distinct from the common features of bullying as mentioned in its definition-power imbalance, repetition, and intentional harm (Olweus, 2013). First, the target of victimization is often publicly identified within the social group (Kwon, 1999). Through the process of collective labeling, everyone in the social group is aware of the victim, which often results in relational ostracism by means of isolation and segregation against the target of victimization (Kwon, 1999). Furthermore, due to such public labeling, victimized youths are often continuously stigmatized with this negative experience even as they move up to the next grade or change schools (Kwon, 1999). Second, the classroom environment provides a rigid structure that perpetuates fixation of victimization roles (Lee et al., 2016). Korean students in secondary schools stay in their home room for all of the courses they take. In a structure in which the same groups of students spend most of their time in the same classroom without meeting peers outside their home room, interpersonal dynamics within the single classroom may play a crucial role in determining victims. Third, the relatively low tolerance for differences and acceptance of diversity among Korean adolescents makes individuals who are "different" stand out (Lee et al., 2016). Thus, anyone who is a source of disruption against group solidarity may likely become a target of peer victimization.

Collective characteristics may also inform the relationship between neighborhood characteristics and bullying experiences. The effect of social context-such as neighborhood factors-on bullying may be more pronounced in collectivist cultures than in individualist cultures due to the strong prominence of group norms in collectivist cultures (Schwartz, 1990; Triandis, Brislin, \& Hui, 1988). In the case of Korea, social cohesion may play an especially important role in reducing adolescent bullying behaviors than formal social control (Cheong, 2009). In detail, the strong bonds and relational network among community members may effectively induce voluntary action to maintain order in the neighborhood, and thereby prevent bullying in the local community (Cheong, 2009). However, as bullying in Korea has been considered an individual-level concern (Hong, Lee, Lee, Lee, \& Garbarino, 2014), the vast majority of studies on bullying experiences in Korea has focused on examining individual- and family-level predictors. This limits our understanding of neighborhood-level predictors of bullying (Park \& Kim, 2015). The unique cultural context of Korea underscores the need to elucidate the different developmental patterns of bullying perpetration and victimization in adolescence, as well as the link between neighborhood-level predictors of such bullying trajectories.

\subsection{Aims and hypotheses}

Although evidence suggests that the developmental patterns of bullying perpetration and victimization experiences may differ by individuals overtime, relatively few studies have used longitudinal data to identify the various trajectories of bullying perpetration and victimization (Juvonen \& Graham, 2014). The few longitudinal studies are limited in using short term data that measured two or three time points, instead of a longer period across adolescence (Reijntjes et al., 2013; Scholte, Engels, Overbeek, De Kemp, \& Haselager, 2007; Seo, 2012), and relying on community samples that are not nationally representative (Reijntjes et al., 2013; Seo, 2012). Furthermore, there is a paucity of research that examines neighborhood predictors to understand the unique trajectories of bullying experience. Research on various trajectories of bullying experience and its neighborhood predictors are especially scarce in non-western contexts, despite the role of unique cultural influences that may shape youth behavior. The current study responds to these gaps with the following study aims: (a) examine latent trajectories of bullying and victimization and (b) identify neighborhood predictors of these trajectories among Korean adolescents. 


\section{2 | METHOD}

\section{1 | Data}

The current study analyzed data from waves 2 to 6 (second year of middle school to third year of high school; years 2011-2015) of the Korean Children and Youth Panel Survey (KCYPS) collected by the National Youth Policy Institute (NYPI). KCYPS is a major longitudinal study on child and adolescent development in South Korea, containing information on children and adolescents' demographic, socioeconomic, and psychological characteristics. One major advantage of the KCYPS is that it contains various items that assess the experience of perpetration and victimization, which is suitable for representing the diverse types of bullying experiences including verbal, physical, and relational bullying among Korean youth (Shin, Jang, \& Cho, 2017). This nationally representative sample of Korean adolescents was selected by multistage stratified cluster sampling, such that the number of sampled students was proportional to the number of student population in 16 administrative districts in Korea (National Youth Policy Institute, 2017a, 2017b). Such research designs that are clustered at the community-level are scarce, despite being ideal for examining neighborhood-effects (Foster \& Brooks-Gunn, 2013). The questionnaire was based on youth self-reports, with the exception of variables measuring family socioeconomic status. Neighborhood information at the Shi/Gun/Gu level (equivalent to census tract in the US) was drawn from the Korean Census (Statistics Korea, 2018a) and official administrative records from the Korean Ministry of Health and Welfare (Ministry of Health \& Welfare, 2012) and the Korean Ministry of Education (Ministry of Education, 2013). Korean Census data is collected from administrative data, web-based surveys, and face-to-face surveys. Data on public assistance, school violence cases, and educational achievement were directly reported by the local government or school officials. Approximately half of the Korean Census data, which includes information on the educational level or structure of household, was collected by web-based survey methods and by face-to-face interviews, respectively. Among the total of 2,351 youths in the KCYPS, our longitudinal analysis was limited to 2,178 youths who had information for at least three time points, the minimum number of time points recommended for longitudinal analysis (Curran, Obeidat, \& Losardo, 2010).

\section{2 | Measures}

\subsection{1 | Dependent variable}

The dependent variable is the trajectory of bullying perpetration (seven items) and victimization (five items) experience derived from wave 2 (2011) to wave 6 (2015) from the KCYPS (National Youth Policy Institute, 2017a, 2017b). Youths responded to seven items that asked if they have had the following bullying perpetration experiences during the past year (e.g., "teasing or mocking," "bullying others," "group fight," "hitting severely," "threatening," "take away money or goods," and "steal money or goods"). This scale originated from the Korean Youth Panel Survey conducted by the NYPI (National Youth Policy Institute, 2017a, 2017b). Youth who answered having experienced at least one of the seven perpetrating behaviors were coded as 1 , and as 0 if otherwise. Experiences of victimization included five items (e.g., "teased or mocked by others," "bullied," "beaten by others," threatened," "took away money or goods by others"; 1 = experienced at least one event of victimization and $0=$ did not experience any victimization).

\subsection{2 | Independent variable}

Individual, family, and neighborhood domains were included in the analyses as independent variables. Individuallevel variables were measured at the youth level (Level 1) at baseline in 2011. All variables were developed or modified from the KCYPS unless indicated otherwise (National Youth Policy Institute, 2017a, 2017b). Male was coded as 1 and 0 if female. Externalizing behavior was the mean of six questions that were modified from research by 
Cho and Lim (2003; e.g., "I tend to find fault with small things," "I tend to fight over nothing," "Sometimes I get angry all day") and Cronbach's $\alpha$ was .81. Internalizing behavior was the average of social withdrawal (Kim \& Kim, 1998; e.g., "I feel a lot of shame," "I am shy," "I hate to be in front of people") and depressive symptoms (Kim, Kim, \& Won, 1984; e.g., "I feel unhappy, sad, or depressed," "I have a lot of worries," "I feel lonely"). Cronbach's $\alpha$ of social withdrawal and depressive symptoms were .85 and .90 , respectively. Externalizing and internalizing behavior were measured on a 4-point Likert scale ( 1 = strongly disagree, 4 = strongly agree), such that a higher score indicated more behavior problems. These individual-level variables were selected based on prior work that identified gender, externalizing, and internalizing behavior as predictors of bullying experiences (Cho, 2013; Lee, Oh, \& Lee, 2014).

Variables in the family domain were measured at the youth level (Level 1) at baseline and were developed or modified from the KCYPS (National Youth Policy Institute, 2017a, 2017b). Two biological parent family was coded as 1 if youth lived with two biological parents, and as 0 for other types of families (e.g., single parent or step parent families). Family income was an open-ended question that measured the level of annual income for the entire family (unit in 10,000,000 Korean Won). Parental education was the highest level of education completed by the father, mother, or other caregivers ( 1 =less than high school, 2 =high school, $3=$ two-year college, $4=$ four-year college, $5=$ advanced degree). Maltreatment was the average of four items on parental abuse (e.g., "When I do something wrong, my parents severely punished me," "My parents have often spoken to me with harsh words or swearing") and another four items on neglect which were coded reversely (e.g., "My parents consider me more important than their work", "My parents are interested in my experiences at school"). These scales were adopted from studies on child maltreatment and parenting by Huh (2000) and Kim (2003). Cronbach's $\alpha$ of abuse and neglect were .77 and .85, respectively. Items in the maltreatment scale were measured on 4-point Likert scale ( $1=$ strongly disagree, $4=$ strongly agree) with a higher value representing more severe maltreatment by parents. Prior research found that these family variables are significant risk factors of victimization and school adjustment among Korean youth (Cho \& Han, 2015; Chung \& Chun, 2012).

All neighborhood variables were measured at the neighborhood-level (Level 2) in which the adolescent's school was located during 2011 unless indicated otherwise. Neighborhood data used in this study were administrated by the Korean Census and Ministry of Education of Korea and contains representative information of each district in Korea. Public assistance (\%) was the proportion of households receiving financial support from the government for housing, education, and medical service in the neighborhood. Low education (\%) was the proportion of households whose primary income earner had completed less than high-school education. Not married (\%) was the proportion of households with non-married status among all households in the district of the youth's school. Low education (\%) and not married (\%) measures at 2011 were interpolated by applying the annual rate of variation between 2010 and 2015, as the Korean Census data is only offered in 5-year intervals. Substance use (per capita) was the average number of respondents who answered that they had drunk or smoked more than once a month during the past year in the neighborhood divided by the total number of individuals in the neighborhood. Official bullying incidents (per capita) was the number of bullying incidents that were referred to the official school council on bullying in the neighborhood divided by the total number of grade-equivalent students in the respective area. Substandard academic performance (per capita) was the number of students whose academic achievement of Korean, English, and Mathematics are below the basic level relative to the total number of students in the region in which the youth's school is located. Collective efficacy was the average of six items that asked youth about the connectedness and affection for the community and its members (e.g., "I know almost everyone in my neighborhood," "I enjoy being with my community members"). Items were measured on a Likert scale and were reverse coded ( $1=$ strongly disagree; 4 = strongly agree), such that higher values represent higher levels of community efficacy. Urbanicity was coded as 1 if youth's school is located in an urban area, and 0 if otherwise. Both collective efficacy and urbanicity were measured at the youth level (Level 1) at baseline year. Moving experience, also individual-level data, was coded as 1 if youth had moved to a different neighborhood at least once over the five waves of data. Collective efficacy, urbanicity, and moving experience were developed or modified by the KCYPS (National Youth Policy Institute, 2017a, 2017b). 


\section{3 | Analytic method}

First, we conducted latent class growth analysis (LCGA) with Mplus (Muthén \& Muthén, 2012), to identify different trajectories of school bullying perpetration and victimization among adolescents over 5 years. LCGA classifies respondents to each latent group considering patterns of individual's response (Muthén \& Muthén, 2000). LCGA suggests the number of latent groups based on several statistical criteria, such as Akaike Information Criterion (AIC), Bayesian Information Criterion (BIC), Sample-size adjusted Bayesian Information Criterion (SSABIC), Entropy, Adjusted Lo-Mendell-Rubin likelihood ratio test (Adj.LRT), Bootstrap Likelihood Ratio Test (BLRT), and sample distribution. A lower information criterion value represents a higher level of model fit (Feldman, Masyn, \& Conger, 2009). Entropy, which indicates the quality of classification, ranges between 0 and 1 , with higher values indicating higher quality of classification (Celeux \& Soromenho, 1996). Adj.LRT and BLRT demonstrate the statistical significance of a model, with a statistically significant $p$ value indicating that the model with $\mathrm{K}$ classes can be selected over the K-1 class model (Feldman et al., 2009; Tein, Coxe, \& Cham, 2013). The classification proportion of each latent class is recommended to be no less than $5 \%$ of the data (Wickrama, Mancini, Kwag, \& Kwon, 2012).

Second, we used STATA 14 (StataCorp., 2015) to employ logistic regression with clustered standard errors at the neighborhood level to examine whether individual, family, and neighborhood variables predicted latent class trajectories. The membership of latent trajectory was a binary variable derived from LCGA. We included three individual, four family, and eight neighborhood variables as predictors of latent trajectories.

Guided by previous research (Shaw, Gilliom, Ingoldsby, \& Nagin, 2003; Singer, \& Willett, 2003), youth with more than three observations in both bullying perpetration and victimization over 5 years were analyzed for LCGA $(n=2,178)$. In the LCGA stage, Full Information Maximum Likelihood (FIML) was used to handle missing values. With the assumption that there is no correlation between missing data and the dependent variable (Galatzer-Levy \& Bonanno, 2012), FIML is a basic method for imputing missing values in longitudinal latent variables models (Newman, 2003; Saunders et al., 2019). Listwise deletion was used when conducting logistic regression $(n=2,021)$.

\section{3 | RESULTS}

\section{1 | Latent trajectories of bullying and victimization}

To identify latent trajectories of bullying perpetration and victimization experience, three information criterion (AIC, BIC, and SSABIC), classification uncertainty (Entropy), two likelihood ratio statistical tests (Adj.LRT and BLRT), and the number of groups less than $5 \%$ of the total cases were considered (Table 1). As the number of groups increased, AIC generally decreased; BIC initially decreased, but increased from the four-class model; SSABIC initially decreased then increased from the six-class model (Figure 1). Entropy ranged from 0.65 to 0.72 across the two-class and six-class models. For the likelihood ratio statistical tests, BLRT was significant in all models, but Adj.LRT was not significant from the four-class model. Class proportions were less than $5 \%$ in models greater than the three-class model. Based on various fit criteria, a three-class model was selected as the final model.

As shown in Figure 2 and Table 2, three patterns of bullying perpetration and victimization trajectories-"lowrisk group" ( $n=1,760,80.8 \%$ ), "transient group" ( $n=290,13.3 \%$ ), "high-risk group" ( $n=128,5.9 \%)$-were identified. Most adolescents belonged to the low-risk group who hardly experienced any bullying perpetration or victimization throughout the 5 years of observation. Individuals in the transient group experienced both bullying perpetration and victimization at the second year of middle school, but unlike the high-risk group, this group is characterized by greater levels of victimization than perpetration and showed a sharp decline after baseline. Compared to the other two trajectories, the high-risk group reported most bullying perpetration and victimization experience over time. This group had the fewest number of adolescents. There was a gradual decrease in bullying experience overtime, but perpetration persisted to be greater than victimization experience throughout all waves. 
TABLE 1 Model fit criteria by number of latent trajectories

\begin{tabular}{lllllllllllllll} 
Class & AIC & BIC & SSABIC & Entropy & Adj.LRT & BLRT & & $\mathbf{1}$ & $\mathbf{2}$ & $\mathbf{3}$ & $\mathbf{4}$ & $\mathbf{5}$ & $\mathbf{6}$ \\
\hline 2 & $7,169.40$ & $7,220.57$ & $7,191.98$ & 0.72 & $<0.001$ & $<0.001$ & 0.89 & 0.11 & & & & \\
\hline 3 & $7,091.36$ & $7,170.97$ & $7,126.49$ & 0.67 & $<0.001$ & $<0.001$ & 0.06 & 0.13 & 0.81 & & & \\
\hline 4 & $7,066.05$ & $7,174.09$ & $7,113.72$ & 0.68 & 0.26 & $<0.001$ & 0.05 & 0.81 & 0.13 & 0.01 & & \\
5 & $7,052.21$ & $7,188.68$ & $7,112.43$ & 0.71 & 0.20 & $<0.001$ & 0.04 & 0.01 & 0.05 & 0.06 & 0.83 & \\
\hline 6 & $7,047.46$ & $7,212.36$ & $7,120.22$ & 0.65 & 0.44 & $<0.05$ & 0.02 & 0.04 & 0.05 & 0.01 & 0.80 & 0.08 \\
\hline
\end{tabular}

Abbreviations: Adj.LRT, adjusted Lo-Mendell-Rubin Likelihood Ratio Test; AIC, Akaike Information Criterion; BIC, Bayesian Information Criterion; BLRT, Bootstrap Likelihood Ratio Test; SSABIC, sample-size adjusted Bayesian Information Criterion.

\section{2 | Descriptive statistics of latent trajectory}

Descriptive statistics of each latent trajectory are presented to illustrate the characteristics of each class before identifying predictive factors (Table 3). The analysis was performed with the data of 2,021 youths who did not have any missing values on the independent variables. Among individual predictors, the proportion of male students was higher in the order of transient (57.4\%), high-risk (55.1\%), and low-risk (49.6\%) groups. Externalizing behavior was highest among the high-risk group and internalizing behavior was highest among the low-risk group.

For family variables, the proportion of adolescents who lived with two biological parents was highest for the low-risk group (13.0\%), followed by the transient group (10.8\%) and the high-risk (9.3\%) group. Income, education, and maltreatment of the high-risk group were higher than those of the low-risk and the transient groups.

In terms of neighborhood variables, public assistance, low education, and collective efficacy in the low-risk group were higher compared to the transient group and the high-risk group. Not married, substance use, official bullying incidents of the high-risk group were higher than those of the other two groups. Substandard academic performance was highest for the transient group. Urbanicity ranged from 80.5\% (low-risk group) to $99 \%$ (high-risk group). Moving experience ranged from $6.8 \%$ (transient group) to $10.8 \%$ (low-risk group).

\subsection{Results of the logistic regression}

Table 4 presents results from logistic regression models with neighborhood clustered standard errors. We conducted three logistic regression models to compare each pair among the three latent trajectories of bullying perpetration and victimization.

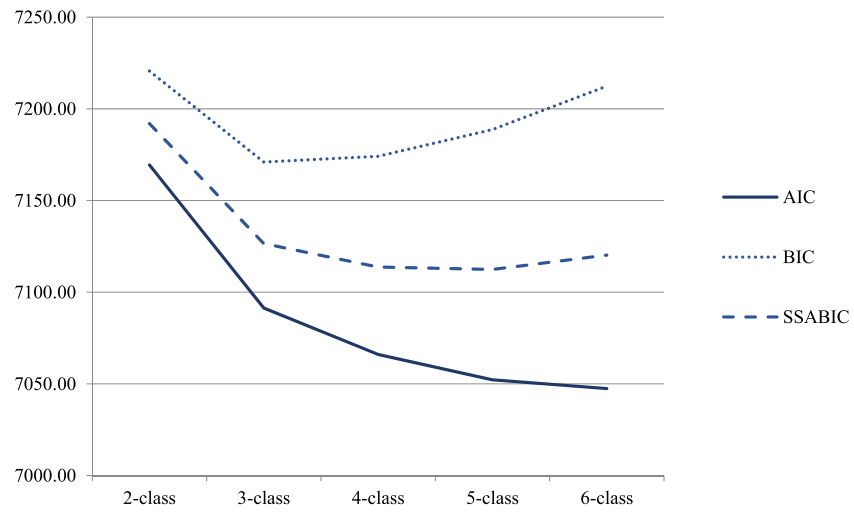

FIGURE 1 Change in information criteria index by number of latent trajectories 
Low-risk group $(n=1,760)$

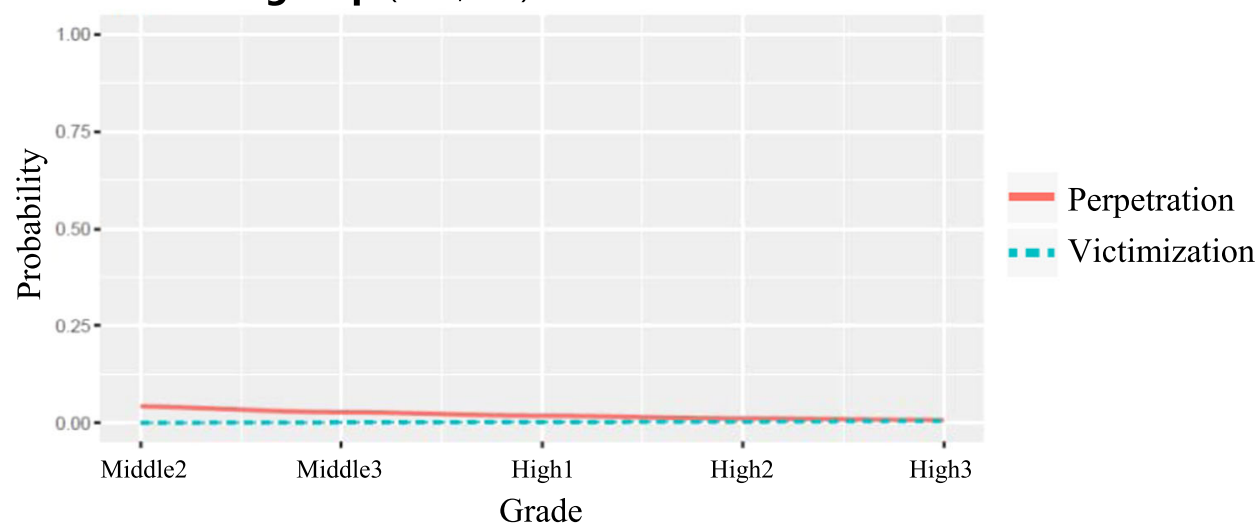

Transient group $(n=290)$

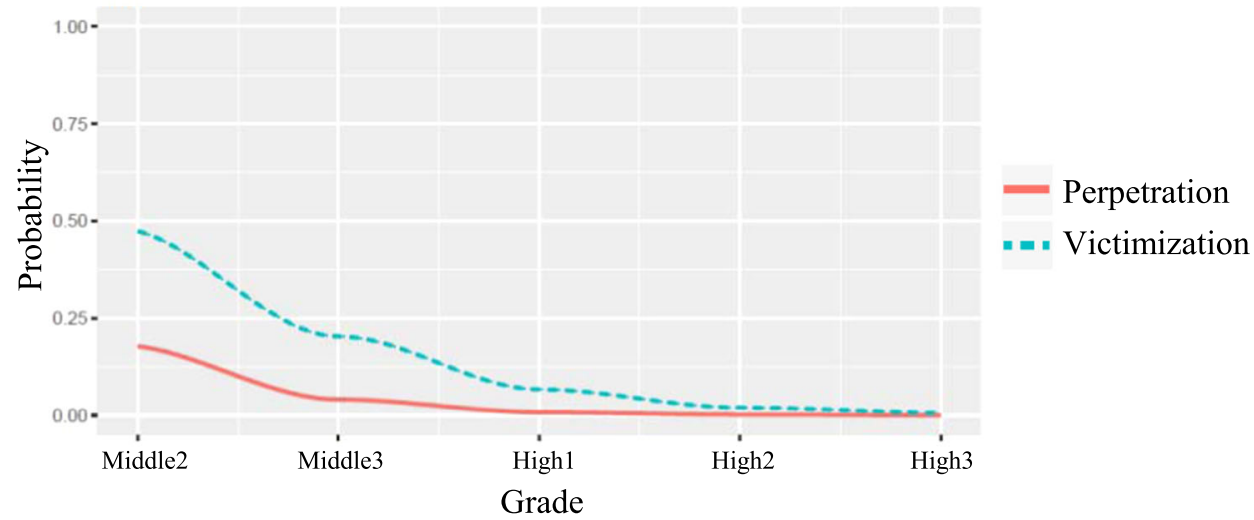

High-risk group $(n=128)$

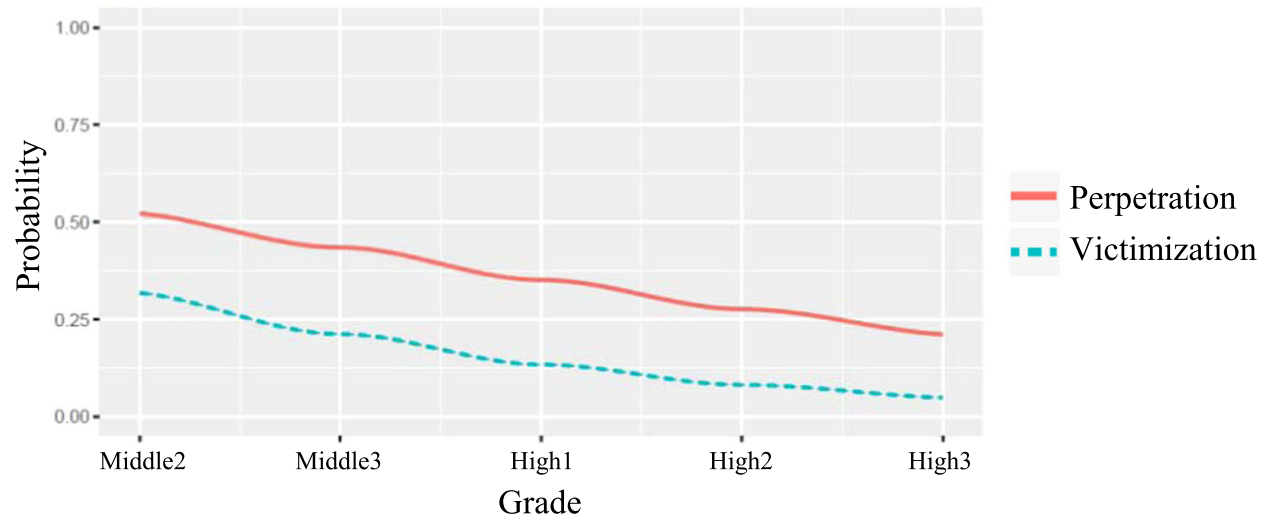

FIGURE 2 Latent trajectories of school bullying $(N=2,178)$

Among individual variables, being male was the only statistically significant predictor between latent groups of adolescent's bullying perpetration and victimization experience. Compared to female adolescents, male adolescents were more likely to be in the transient group than the low-risk group $(\beta=.34, p<.05)$. No family variables were significant predictors for the latent trajectories of bullying perpetration and victimization experience of adolescents. 
TABLE 2 Probability of three latent trajectories

\begin{tabular}{|c|c|c|c|c|c|c|}
\hline \multirow[b]{3}{*}{ Class } & \multicolumn{6}{|c|}{ Probability ( $p$ value) } \\
\hline & \multicolumn{3}{|c|}{ Perpetration } & \multicolumn{3}{|c|}{ Victimization } \\
\hline & Low-risk & Transient & High-risk & Low-risk & Transient & High-risk \\
\hline Middle2 & $\begin{array}{l}0.04 \\
(0.00)^{* * *}\end{array}$ & $\begin{array}{l}0.18 \\
(0.00)^{* * *}\end{array}$ & $\begin{array}{l}0.52 \\
(0.00)^{* * *}\end{array}$ & $\begin{array}{l}0.00 \\
(0.46)\end{array}$ & $\begin{array}{l}0.47 \\
(0.00)^{* * *}\end{array}$ & $\begin{array}{l}0.32 \\
(0.00)^{* * *}\end{array}$ \\
\hline Middle3 & $\begin{array}{l}0.03 \\
(0.00)^{* * *}\end{array}$ & $\begin{array}{l}0.04 \\
(0.06)\end{array}$ & $\begin{array}{l}0.44 \\
(0.00)^{* * *}\end{array}$ & $\begin{array}{l}0.00 \\
(0.34)\end{array}$ & $\begin{array}{l}0.20 \\
(0.00)^{* * *}\end{array}$ & $\begin{array}{l}0.21 \\
(0.00)^{* * *}\end{array}$ \\
\hline High1 & $\begin{array}{l}0.02 \\
(0.00)^{* * *}\end{array}$ & $\begin{array}{l}0.01 \\
(0.32)\end{array}$ & $\begin{array}{l}0.35 \\
(0.00)^{* * *}\end{array}$ & $\begin{array}{l}0.00 \\
(0.18)\end{array}$ & $\begin{array}{l}0.07 \\
(0.00)^{* * *}\end{array}$ & $\begin{array}{l}0.13 \\
(0.00)^{* * *}\end{array}$ \\
\hline High2 & $\begin{array}{l}0.01 \\
(0.00)^{* * *}\end{array}$ & $\begin{array}{l}0.00 \\
(0.49)\end{array}$ & $\begin{array}{l}0.28 \\
(0.00)^{* * *}\end{array}$ & $\begin{array}{l}0.00 \\
(0.05)^{*}\end{array}$ & $\begin{array}{l}0.02 \\
(0.00)^{* *}\end{array}$ & $\begin{array}{l}0.08 \\
(0.00)^{* *}\end{array}$ \\
\hline High3 & $\begin{array}{l}0.01 \\
(0.00)^{* * *}\end{array}$ & $\begin{array}{l}0.00 \\
(0.60)\end{array}$ & $\begin{array}{l}0.21 \\
(0.00)^{* * *}\end{array}$ & $\begin{array}{l}0.01 \\
(0.02)^{*}\end{array}$ & $\begin{array}{l}0.01 \\
(0.04)^{*}\end{array}$ & $\begin{array}{l}0.05 \\
(0.02)^{*}\end{array}$ \\
\hline
\end{tabular}

${ }^{*} p<.05$

${ }^{* *} p<.01$.

${ }^{* * *} p<.001$

A number of neighborhood variables significantly predicted the latent trajectories of bullying perpetration and victimization experiences among adolescents. Adolescents who lived in neighborhoods with higher portion of households receiving public assistance were more likely to be in the high-risk group than the low-risk group $(\beta=1.12, p<.05)$ and the transient group $(\beta=1.41, p<.05)$. Adolescents in neighborhoods with higher proportion of not married families were also more likely to be in the high-risk group than the low-risk group $(\beta=.24, p<.05)$ and the transient group $(\beta=.18, p<.10)$. As official bullying incidents in the neighborhood increased, adolescents were

TABLE 3 Descriptive summary by latent trajectory $(N=2,021)$

\begin{tabular}{|c|c|c|c|c|c|c|}
\hline \multirow[b]{2}{*}{ Variables } & \multicolumn{2}{|c|}{$\begin{array}{l}\text { Low-risk (a) } \\
n=1,626\end{array}$} & \multicolumn{2}{|c|}{$\begin{array}{l}\text { Transient (b) } \\
n=277\end{array}$} & \multicolumn{2}{|c|}{$\begin{array}{l}\text { High-risk (c) } \\
n=118\end{array}$} \\
\hline & M & $S D$ & M & $S D$ & M & $S D$ \\
\hline \multicolumn{7}{|l|}{ Individual variables } \\
\hline Male (=1) & \multicolumn{2}{|c|}{$807(49.6 \%)$} & \multicolumn{2}{|c|}{159 (57.4\%) } & \multicolumn{2}{|c|}{65 (55.1\%) } \\
\hline Externalizing behavior & 2.13 & 0.57 & 2.10 & 0.58 & 2.14 & 0.64 \\
\hline Internalizing behavior & 2.04 & 0.57 & 2.01 & 0.57 & 1.97 & 0.54 \\
\hline \multicolumn{7}{|l|}{ Family variables } \\
\hline Two biological parent family $(=1)$ & \multicolumn{2}{|c|}{$212(13.0 \%)$} & \multicolumn{2}{|c|}{$30(10.8 \%)$} & \multicolumn{2}{|c|}{$11(9.3 \%)$} \\
\hline Family income & 4.46 & 2.36 & 4.78 & 2.84 & 5.08 & 3.13 \\
\hline Parental education & 2.99 & 1.07 & 3.02 & 1.05 & 3.33 & 1.09 \\
\hline Maltreatment & 1.84 & 0.47 & 1.84 & 0.47 & 1.93 & 0.56 \\
\hline \multicolumn{7}{|l|}{ Neighborhood variables } \\
\hline Public assistance (\%) & 3.14 & 1.30 & 2.99 & 1.26 & 3.07 & 1.41 \\
\hline Low education (\%) & 28.76 & 11.99 & 27.53 & 11.15 & 21.39 & 11.27 \\
\hline Not married (\%) & 7.67 & 1.82 & 7.73 & 1.68 & 7.95 & 1.75 \\
\hline Substance use (per capita) & 41.01 & 3.12 & 41.07 & 2.87 & 41.81 & 1.86 \\
\hline Official bullying incidents (per capita) & 0.88 & 2.32 & 1.83 & 3.84 & 7.31 & 4.78 \\
\hline Substandard academic performance (per capita) & 2.19 & 0.70 & 2.22 & 0.77 & 2.19 & 0.70 \\
\hline Collective efficacy & 2.25 & 0.54 & 2.21 & 0.60 & 2.22 & 0.55 \\
\hline Urbanicity $(=1)$ & \multicolumn{2}{|c|}{$1356(80.5 \%)$} & \multicolumn{2}{|c|}{$240(86.6 \%)$} & \multicolumn{2}{|c|}{$117(99 \%)$} \\
\hline Moving experience $(=1)$ & \multicolumn{2}{|c|}{$176(10.8 \%)$} & \multicolumn{2}{|c|}{$8(6.8 \%)$} & \multicolumn{2}{|c|}{26 (9.4\%) } \\
\hline
\end{tabular}

Abbreviations: $M$, mean; $S D$, standard deviation. 
expected to fall into the high-risk group than the low-risk $(\beta=.48, p<.001)$ and transient group $(\beta=.37, p<.01)$, and they also were expected to fall into the transient group than the low-risk group $(\beta=.11, p<.001)$. Adolescents who reported higher levels of collective efficacy were less likely to be in the high-risk group than the low-risk group $(\beta=-.30, p<.05)$. When compared with adolescents who have not moved to different neighborhoods, adolescents who moved during wave 2 to wave 6 were less likely to be in the high-risk group than the low-risk group $(\beta=-1.15$, $p<.05)$. However, low education, substance use, substandard academic performance, and urbanicity were not significant predictors of the latent trajectories of bullying perpetration and victimization of adolescents.

\section{4 | DISCUSSION}

Bullying perpetration and victimization is a pervasive phenomenon among youth around the globe that has detrimental effects on emotional and behavioral outcomes that extend beyond childhood (Barker, Arseneault, Brendgen, Fontaine, \& Maughan, 2008; Kretschmer, Veenstra, Deković, \& Oldehinkel, 2017; Nansel et al., 2004). This study examined various developmental trajectories of bullying perpetration and victimization experiences, as well as the individual, family, and neighborhood antecedents of these longitudinal paths. Using a latent class growth analysis approach, we identified three unique trajectories of bullying perpetration and victimization experience in adolescence. In addition, we found several neighborhood factors that predict these distinct developmental paths.

\section{1 | Low-risk, transient, and high-risk trajectories}

Study findings revealed three different trajectories of bullying perpetration and victimization experience. In the current study, the vast majority of youth were in the low-risk group, who experienced no to minimal bullying perpetration and victimization throughout the 5 years of observation. This finding is in line with prior longitudinal studies that have reported that the low-risk group is most prevalent compared to other types of bullying experiences. Specifically, in a study of Scottish youth, $75 \%$ of the sample belonged to the group with minimal perpetration and victimization experience (Barker et al., 2008). For bullying perpetration, Reijntjes et al. (2013) showed that the low bullying trajectory comprised $82 \%$ of the study sample among fourth to sixth graders in the Netherlands. Similar results were derived from longitudinal observations of victimization with the non-victim group being the vast majority at $88 \%$ among Canadian youth in grades 5 to 7 (Goldbaum, Craig, Pepler, \& Connolly, 2003). In a nationally representative data of Korean adolescents who were followed from fifth grade of elementary school to second grade of middle school, the lowest victimization group comprised $82 \%$ of the study sample (Ahn, 2018).

Our finding that the group with the least experience of bullying perpetration and victimization trajectories comprises the majority, however, should not distract public attention away from the aversive bullying phenomenon. The low frequency of bullying does not equate with the decline in harm or intensity of bullying (Hong, No, \& Lee, 2011). Individuals who are involved-even to a small extent-seem to experience detrimental outcomes, compared to non-involved individuals (Goldbaum et al., 2003). Furthermore, despite the declining trend, youth who have had prior history of bullying perpetration or victimization may be at greater risk of experiencing risk behaviors in the future (Kretschmer et al., 2017). Particularly in Korea, there is evidence that among individuals who are involved in bullying, their experiences have become more violent and that the consequences of bullying seem to be more severe than youth in the past. Recent statistics show that about $10.4 \%$ of the reported bullies were sent to the Juvenile Court in 2018, which was a 16.6\% increase compared to 2017 (Korean National Police Agency, 2018). Further, a recent study of bullying trends in Korea found an increase in insult and defamation incidents, which are psychologically devastating for the victim (Kim, Kim, \& Son, 2018). Low prevalence, therefore, should not minimize the scope of the bullying phenomenon.

Similarities between the transient and high-risk groups lie in the relatively higher prevalence of bullying perpetration and victimization than the low-risk group at baseline (second year of middle school), which then 

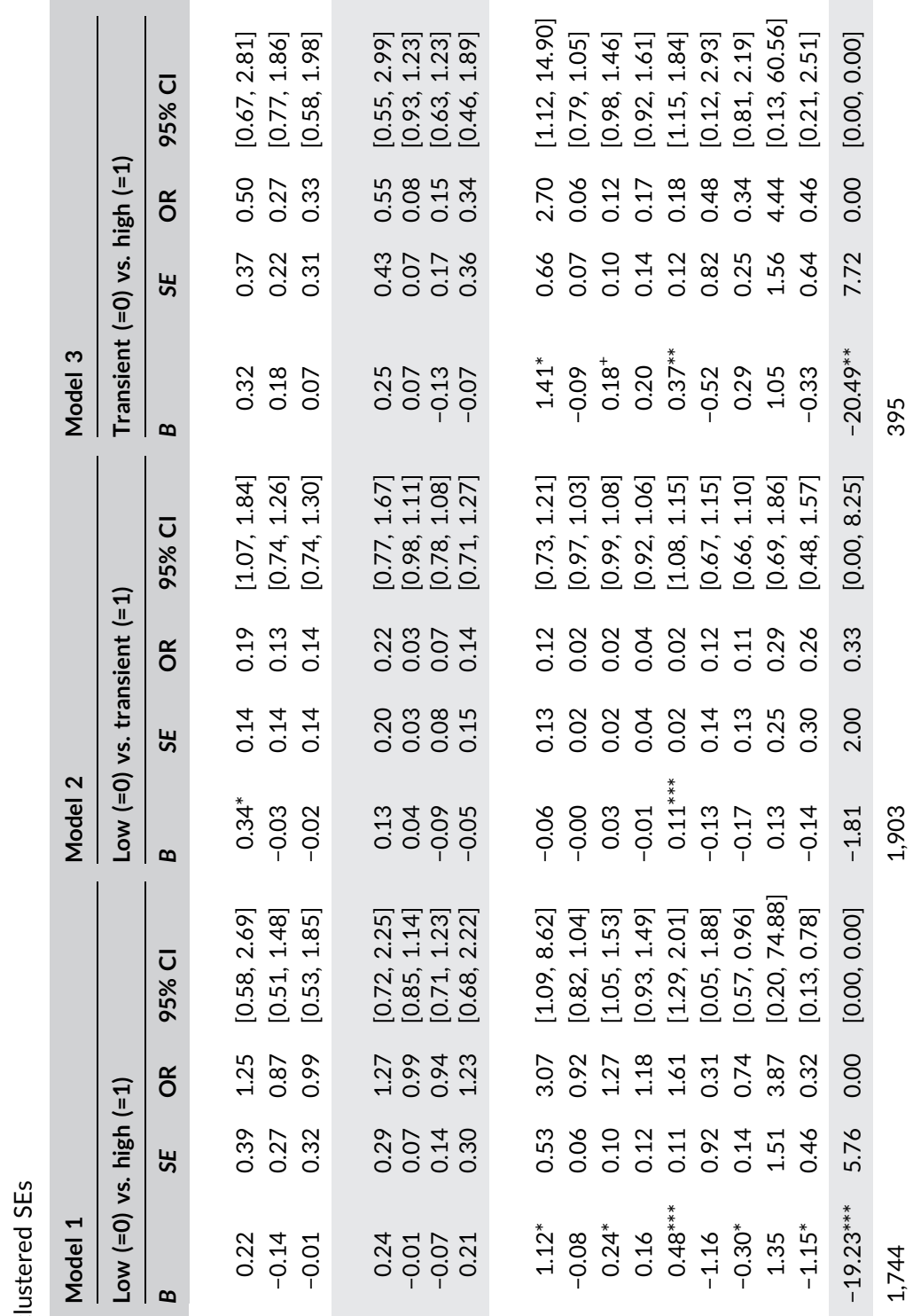

๙ ก क ने लं 응ㅇ 응ㅇㅇㅇ

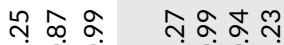

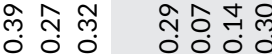

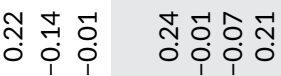

तु

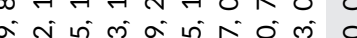

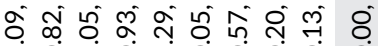

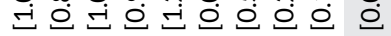

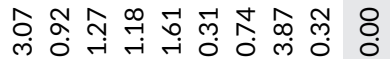

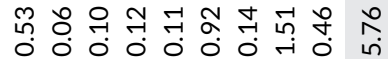

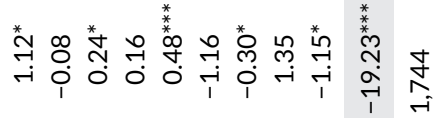
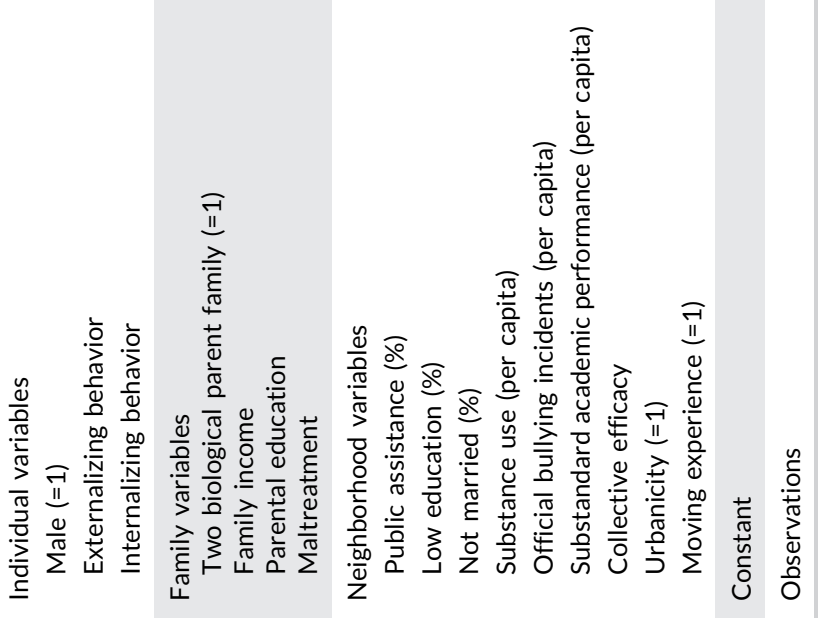

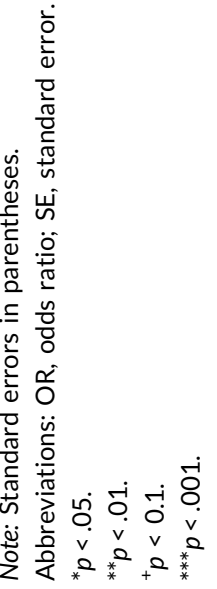


showed a declining trend. These results are consistent with the general longitudinal pattern that depicts bullying experience in adolescence-there is an increase in bullying involvement starting in the later years of elementary school, which peaks in middle school, and then declines afterward (Ahn, 2018; Barker et al., 2008; Han et al., 2016; Kretschmer et al., 2017; No, Lee, Lee, \& Hong, 2017). From a social dominance perspective, entry to middle school marks a period of social status formation, during which explicit and implicit forms of aggression is used to maintain or reconstruct an individual's social dominance position (Pellegrini \& Long, 2002). Variation in the timing of pubertal maturity and expansion of social peer groups (Craig, Pepler, Connolly, \& Henderson, 2001), as well as the egocentric redirection toward the self (Elkind, 1967) during early middle school years may reinforce the need for reorganization of such social hierarchies. Thus, the peak in bullying perpetration and victimization seen at the beginning of the current study may be explained as the use of aggressive strategies among adolescents to acquire a higher social status among peers. However, as the dominance relationships are gradually negotiated and stabilized over time, individuals may be less likely to engage in bullying (Pellegrini \& Long, 2002). Furthermore, in a culture such as Korea where adolescents are especially concerned about education and occupational success (Statistics Korea, 2018b), entry to a prestigious college may become the foremost important agenda in high school, such that gaining dominance through aggressive means may become less desirable during later years of adolescence than in mid-adolescence.

An interesting observation from the three trajectories is that bullying perpetration and victimization tend to occur together, rather than exhibiting a trajectory of pure-bullies or pure-victims. Specifically, the transient and high risk groups showed a declining trend for both perpetration and victimization experiences. Similarities in the longitudinal change for perpetration and victimization experiences point to bully-victims among various bullyparticipant roles (Salmivalli, Lagerspetz, Björkqvist, Österman, \& Kaukiainen, 1996). Compared to pure bullies or pure victims, bully-victims are more vulnerable as they share the negative experiences of pure-bullies and purevictims (Lester, Cross, Shaw, \& Dooley, 2012). Specifically, bully-victims show higher levels of internalizing and externalizing behaviors (Nansel et al., 2004). These negative experiences and characteristics place victims at a more vulnerable position, which possibly makes them most sensitive to individual and neighborhood environments; hence, bully-victims require most attention and resources. The present study is unique and advances the literature in that it captured the coexistence of bullying perpetration and victimization. This comorbidity of perpetration and victimization is not detected in most studies that examined developmental paths of only perpetrating (Hong et al., 2011; Kretschmer et al., 2017) or victimization (Ahn, 2018; Chang, 2013; Goldbaum et al., 2003) experiences.

However, the transient and high-risk groups differ in terms of speed of decline and prevalence of perpetration and victimization experiences. The transient group is a primarily victimized group, who also engage in some level of bullying perpetration mostly during the second year of middle school. For the transient group, both perpetration and victimization experiences decline to almost non-involvement-similar to the low-risk group-by the end of high school. The high-risk group, however, experience both bullying perpetration and victimization that is followed by a steadily decline. Although bullying experiences decline over time for many youth, youth in this high-risk group may still rely on bullying as a goal-directed behavior with means of resource control and regulation (Volk, Dane, \& Marini, 2014).

\subsection{Neighborhood predictors distinguish bullying trajectories}

As indicated by the ecological framework, our findings suggest the important role of neighborhood-level factors in predicting trajectories of bullying perpetration and victimization experience. In particular, consistent with the tenets of collective efficacy theory, living in neighborhoods with lower levels of social cohesion and trust predicted increased likelihood of being in the high-risk group, compared to the low-risk and transient groups. Youth residing in neighborhoods that lack social cohesion and trust are likely to observe more frequent perpetration and victimization acts in their communities than their counterparts in socially cohesive neighborhoods. Thus, it is plausible that exposure to prevalent interpersonal violence and the absence of a cohesive community-level 
intervention may have left youth to consider bullying perpetration as acceptable and normative behavior. Furthermore, consistent with social disorganization theory, the proportion of families receiving public assistance, rates of unmarried families, and officially reported cases of per capita bullying incidents were linked with greater probability of being in the high-risk group, compared to membership in the low-risk and transient group. There was little difference between the low-risk group and the transient group, however, with the officially reported cases of per capita bullyings incidents being the only statistically significant neighborhood-level predictor. These results extend existing research by confirming the associations of disadvantaged structural conditions and disorganized neighborhood contexts that surround adolescents with bullying perpetration and victimization experience (Cook et al., 2010; Leventhal \& Brooks-Gunn, 2000).

An unexpected finding from the present study was that individual-level factors did not significantly predict membership in bullying trajectories, with the exception of male youth increasing the likelihood of being in the transient group, compared to the low-risk group. Although very scarce, studies that examined neighborhood predictors of bullying trajectories using latent growth curve models have reported similar results. For example, Kretschmer et al. (2017) found a link between individual and family factors and initial bullying experience, but evidence for these variables as predictors of longitudinal trends were limited. Similarly, in a multilevel study by No et al. (2017), individual-level variables were salient predictors of initial levels of bullying perpetration and victimization, whereas school-level variables were significant predictors of the slope of growth trajectories of bullying perpetration and victimization.

Our findings highlight the importance of neighborhood contexts in predicting longitudinal trends of bullying perpetration and victimization in adolescence. A plethora of research has found the deleterious influence of chronic exposure to environmental stressors on youth development. For instance, several studies show the relationship between environmental factors and increased levels of stress and behavioral problems (Ross, 2000; Ross, Reynolds, \& Geis, 2000; Xue et al., 2005). Further, neighborhood contexts of individuals' residential area, such as aggregated socioeconomic characteristics (Silver, Mulvey, \& Swanson, 2002) and perception of incivilities (Ross, 2000) were found to predict psychological maladaptation of its residents. Another plausible explanation can be made upon the life-course perspective of risk behavior. A study revealed that ecological disadvantages of neighborhoods are related to prosocial competence, social integration, and differential associations with peers (Elliott et al., 1996). A longitudinal study that tracks the development of a nationally representative birth cohort of 2,232 British children found that the relationship between neighborhood factors and children's antisocial behavior was strengthened across childhood (Odgers et al., 2012).

\section{3 | Limitation}

Several limitations should be considered when interpreting the analytic results. First, the neighborhood characteristics in the current study describe the features at the administrative level (i.e., Shi/Gun/Gu level) in which the youth's school is located. Youth whose daily routine activities involve two or more neighborhood contexts may in fact be exposed to various neighborhood environments-however, this possibility is not captured in our study. Furthermore, neighborhood boundaries that are objectively defined for administrative purposes may not accurately represent youths' subjective understanding of their neighborhood contexts that strongly predict behavioral outcomes (Campbell, Henly, Elliott, \& Irwin, 2009). Another limitation is that the measures of bullying perpetration and victimization were limited to mostly verbal and physical aggression. As exposure to cyberbullying-an increasingly common form of aggression among adolescents-may lead to different trajectories (Hinduja \& Patchin, 2008; Jang, Song, \& Kim, 2014), the relationship between cyberbullying and victimization should be considered in future research. Due to limited availability of neighborhood-level data, the current study was not able to consider neighborhood factors that may be associated with bullying experiences. Future studies may benefit from incorporating other community-level factors, such as income inequality (Elgar, Craig, Boyce, Morgan, \& Vella-Zarb, 2009), violence exposure (Khoury-Kassabri et al., 2004; Low \& Espelage, 2014), and availability or accessibility of high quality institutional resources (Leventhal \& Brooks-Gunn, 2000). Additionally, analysis utilized the average level of perpetration and victimization experienced by youth. As specific types of perpetration and victimization may be more salient at a particular age or a pubertal development stage (Craig et al., 2001), research that encompass a 
broader range of bullying experiences is warranted. Finally, our study did not specifically test how bullying perpetration and victimization trajectories are formed. In other words, the exact mechanisms that shape the different trajectories were not specifically tested. Questions such as "Which factors explain the steeper decline for the transient group as compared to the high-risk group?" or "Which unique features of the high school period, compared to the middle school period contribute to reducing bullying involvement?" should be explored in future studies as examination of specific risk or protective processes at various ecological levels that link neighborhood characteristics and bullying experience may provide malleable areas for targeted intervention (Low \& Espelage, 2014; Schmidt et al., 2016).

\subsection{Implications for research and practice}

To date, bullying has been generally considered as an individual-level phenomenon (Hong et al., 2014) as shown in the theoretical basis used in the Korean literature, which highlights psychological and individual predictors of bullying (Park \& Kim, 2015). The few studies that have considered contextual factors are limited by conceptualizing and modeling neighborhood predictors as control variables or considering them of secondary importance relative to individual-level factors (Park \& Kim, 2015). Naturally, intervention efforts against bullying have focused on individual-level psychological traits and behaviors (Hong et al., 2014).

The current study findings, however, provide empirical support that neighborhood factors are in fact, of importance in understanding developmental changes in bullying perpetration and victimization. Specifically, disadvantaged structural conditions, such as public assistance, family structure, and officially reported cases of bullying, and process-oriented mechanisms, such as collective efficacy, were strong predictors of bullying perpetration and victimization experience in adolescence. Such findings lend support to the view that the most effective prevention and intervention strategies against bullying perpetration and victimization in the Korean context would be those that consider multiple domains of the ecological system (Hong et al., 2014).

\section{5 | CONCLUSION}

The current study advances the existing bullying literature by identifying distinct developmental profiles of bullying perpetration and victimization. Joint trajectories of perpetration and victimization can inform social service or policy decisions as each developmental path may represent unique experiences among youth in need of specific resources for treatment or intervention. Furthermore, an in-depth understanding of these paths is important as the cumulative effects of persistent or instable perpetration or victimization experiences may have various detrimental effects on future outcomes (Goldbaum et al., 2003). This study also addresses the lack of research on neighborhood-level predictors and identifies several important neighborhood indicators that predict developmental trajectories of perpetration and victimization among adolescents.

\section{ACKNOWLEDGMENT}

This study was supported by the Ministry of Education of the Republic of Korea and the National Research Foundation of Korea (NRF-2016S1A5A2A03925702).

\section{ORCID}

Yoonsun Han iD http://orcid.org/0000-0002-1108-0275

Hayoung Kim iD http://orcid.org/0000-0002-5633-1488

Julie Ma iD http://orcid.org/0000-0003-4644-6262

Juyoung Song (iD http://orcid.org/0000-0002-5077-363X

Hyunhee Hong (D) http://orcid.org/0000-0001-6934-2584 


\section{REFERENCES}

Ahn, T. (2018). The developmental trajectories of school violence victimization experience, the related predictors and outcomes: A longitudinal study with applied GMM. The Korean Journal of Elementary Counseling, 17(1), 81-101.

Barker, E. D., Arseneault, L., Brendgen, M., Fontaine, N., \& Maughan, B. (2008). Joint development of bullying and victimization in adolescence: Relations to delinquency and self-harm. Journal of the American Academy of Child \& Adolescent Psychiatry, 47(9), 1030-1038. https://doi.org/10.1097/CHI.ObO13e31817eec98

Bronfenbrenner, U. (1979). The ecology of human development: Experiments by nature and design. Cambridge, MA: Harvard University Press.

Campbell, E., Henly, J. R., Elliott, D. S., \& Irwin, K. (2009). Subjective constructions of neighborhood boundaries: Lessons from a qualitative study of four neighborhoods. Journal of Urban Affairs, 31(4), 461-490. https://doi.org/10.1111/j. 1467-9906.2009.00450.x

Celeux, G., \& Soromenho, G. (1996). An entropy criterion for assessing the number of clusters in a mixture model. Journal of Classification, 13(2), 195-212. https://doi.org/10.1007/BF01246098

Chang, A. (2013). The developmental trajectories of school violence victimization. Journal of Korean Criminological Association, 7(1), 61-82.

Cheong, J. (2009). A study on the cause of school violence: Focusing on the effect of neighborhood characteristics. Korean Association of Public Safety and Criminal Justice Review, 35, 364-394.

Cho, B., \& Lim, K. (2003). Development and validation of emotional or behavioral problems scale. The Korean Journal of Counseling and Psychotherapy, 15(4), 729-746.

Cho, Y. (2013). Gender differences in prevalence and predictors of school violence. Journal of Adolescent Welfare, 15(1), $155-179$.

Cho, Y., \& Han, S. (2015). An analysis of student and parent factors influencing school adjustment. The Journal of Education Assignment Institute, 21(2), 117-144.

Chung, H., \& Chun, J. (2012). Risk factors of adolescents' victimization by school violence. Journal of Adolescent Welfare, 14(1), 195-212.

Cook, C. R., Williams, K. R., Guerra, N. G., Kim, T. E., \& Sadek, S. (2010). Predictors of bullying and victimization in childhood and adolescence: A meta-analytic investigation. School Psychology Quarterly, 25(2), 65-83. https://doi.org/10.1037/ a0020149

Copeland, W. E., Wolke, D., Angold, A., \& Costello, E. J. (2013). Adult psychiatric outcomes of bullying and being bullied by peers in childhood and adolescence. JAMA Psychiatry, 70(4), 419-426. https://doi.org/10.1001/jamapsychiatry.2013. 504

Corvo, K. (2010). Towards an integrated theory of relational violence: Is bullying a risk factor for domestic violence? Aggression and Violent Behavior, 15(3), 181-190. https://doi.org/10.1016/j.avb.2009.12.001

Craig, W. M., Pepler, D. J., Connolly, J., \& Henderson, K. (2001). Developmental context of peer harassment in early adolescence. In J. Juvonen, \& S. Graham (Eds.), Peer harassment in school: The plight of the vulnerable and victimized (pp. 242-261). New York, NY: Guilford Press.

Crick, N. R., \& Dodge, K. A. (1996). Social information-processing mechanisms in reactive and proactive aggression. Child Development, 67(3), 993-1002. https://doi.org/10.2307/1131875

Curran, P. J., Obeidat, K., \& Losardo, D. (2010). Twelve frequently asked questions about growth curve modeling. Journal of Cognition and Development, 11(2), 121-136.

Elgar, F. J., Craig, W., Boyce, W., Morgan, A., \& Vella-Zarb, R. (2009). Income inequality and school bullying: Multilevel study of adolescents in 37 countries. Journal of Adolescent Health, 45(4), 351-359.

Elkind, D. (1967). Egocentrism in adolescence. Child Development, 38(4), 1025-1034. https://doi.org/10.2307/1127100

Elliott, D. S., Wilson, W. J., Huizinga, D., Sampson, R. J., Elliott, A., \& Rankin, B. (1996). The effects of neighborhood disadvantage on adolescent development. Journal of Research in Crime and Delinquency, 33(4), 389-426. https://doi.org/ $10.1177 / 0022427896033004002$

Espelage, D. L., \& Swearer, S. M. (2003). Research on school bullying and victimization: What have we learned and where do we go from here? School psychology review, 32(3), 365-384.

Espelage, D. L., \& Swearer, S. M. (2009). Contributions of three social theories to understanding bullying perpetration and victimization among school-aged youth. In Harris, M. (Ed.), Bullying, rejection, and peer victimization: A social cognitive neuroscience perspective (pp. 151-170). New York, NY: Springer Publishing Company.

Feldman, B. J., Masyn, K. E., \& Conger, R. D. (2009). New approaches to studying problem behaviors: A comparison of methods for modeling longitudinal, categorical adolescent drinking data. Developmental Psychology, 45(3), 652-676. https://doi.org/10.1037/a0014851

Foster, H., \& Brooks-Gunn, J. (2013). Neighborhood, family and individual influences on school physical victimization. Journal of Youth and Adolescence, 42(10), 1596-1610. 
De Frutos, T. H. (2013). Five independents variables affecting bullying: Neighborhood, family, school, gender-age and mass media. Sociology Mind, 3(4), 304-313. https://doi.org/10.4236/sm.2013.34041

Galatzer-Levy, I. R., \& Bonanno, G. A. (2012). Beyond normality in the study of bereavement: Heterogeneity in depression outcomes following loss in older adults. Social Science \& Medicine, 74(12), 1987-1994. https://doi.org/10.1016/j. socscimed.2012.02.022

Goldbaum, S., Craig, W. M., Pepler, D., \& Connolly, J. (2003). Developmental trajectories of victimization: Identifying risk and protective factors. Journal of Applied School Psychology, 19(2), 139-156. https://doi.org/10.1300/J008v19n02_09

Han, Y., Lee, H., Kim, S., \& Lee, Y. (2016). An analysis of mobility of experiencing school violence and the influential actors. Asian Journal of Education, 17(1), 145-168. https://doi.org/10.15753/aje.2016.03.17.1.145

Hinduja, S., \& Patchin, J. W. (2008). Cyberbullying: An exploratory analysis of factors related to offending and victimization. Deviant Behavior, 29(2), 129-156. https://doi.org/10.1080/01639620701457816

Hong, J., Lee, C., Lee, J., Lee, N., \& Garbarino, J. (2014). A review of bullying prevention and intervention in South Korean schools: An application of the social-ecological framework. Child Psychiatry \& Human Development, 45(4), 433-442.

Hong, J. S., \& Eamon, M. K. (2009). An ecological approach to understanding peer victimization in South Korea. Journal Of Human Behavior In The Social Environment, 19(5), 611-625.

Hong, J. S., \& Espelage, D. L. (2012). A review of research on bullying and peer victimization in school: An ecological system analysis. Aggression and Violent Behavior, 17(4), 311-322. https://doi.org/10.1016/j.avb.2012.03.003

Hong, S., No, U., \& Lee, H. (2011). A semi-parametric group-based approach to analyzing latent classes in adolescents' violence frequency trajectories. Studies on Korean Youth, 22(3), 175-195.

Huh, M. (2000). The study for the development and validation of parenting behavior inventory' perceived by adolescent. (Doctoral dissertation). The Graduate School of Ewha Womans University, Seoul.

Jang, H., Song, J., \& Kim, R. (2014). Does the offline bully-victimization influence cyberbullying behavior among youths? Application of general strain theory. Computers in Human Behavior, 31, 85-93. https://doi.org/10.1016/j.chb.2013.10.007

Juvonen, J., \& Graham, S. (2014). Bullying in schools: The power of bullies and the plight of victims. Annual Review of Psychology, 65, 159-185. https://doi.org/10.1146/annurev-psych-010213-115030

Khoury-Kassabri, M., Benbenishty, R., Avi Astor, R., \& Zeira, A. (2004). The contributions of community, family, and school variables to student victimization. American Journal of Community Psychology, 34(3-4), 187-204. https://doi.org/10. 1007/s10464-004-7414-4

Kim, C. (2018). Structural relationships between school violence, family resilience, hopelessness, depression, and suicidal ideation in adolescents. Journal of the Korean Society of Child Welfare, 61, 51-80. https://doi.org/10.24300/jkscw.2018.3.61.51

Kim, H., Kim, C., \& Son, E. (2018). A study on the current status and responsive measures of school violence and professional counseling teacher. Journal of Learner-Centered Curriculum and Instruction, 18(18), 379-405.

Kim, K., Kim, J., \& Won, H. (1984). Korean manual of symptom checklist-90-revision. Seoul: Chungang Aptitude Publishing.

Kim, M., Hong, S., \& Jung, Y. (2017). Factors affecting bullying victimization in school among upper grade of elementary school students in the Jeju area: Focus on depressive symptoms and family-related factors. Anxiety and Mood, 13(1), 25-31.

Kim, M. J., Catalano, R. F., Haggerty, K. P., \& Abbott, R. D. (2011). Bullying at elementary school and problem behaviour in young adulthood: A study of bullying, violence and substance use from age 11 to age 21. Criminal Behaviour and Mental Health, 21(2), 136-144. https://doi.org/10.1002/cbm.804

Kim, S. (2003). The effect of social support on abused children's adjustment (Master thesis) Seoul National University, Seoul.

Kim, S., \& Kim, K. (1998). Development of behavior problem scale for children and adolescence. Journal of Korean Home Management Association, 16(4), 155-166.

Kim, Y., \& Hong, K. (2018). The effects of violence experience in middle school on violence experience and peer relationship in high school. The Journal of Humanities and Social Science, 9(5), 1413-1426.

Koo, H., Kwak, K., \& Smith, P. K. (2008). Victimization in Korean schools: The nature, incidence, and distinctive features of Korean bullying or wang-ta. Journal of School Violence, 7(4), 119-139. https://doi.org/10.1080/15388220801974084

Korean National Police Agency. (2018). Analysis of juvenile delinquency in 2018. Seoul, South Korea: Women and Adolescents Department.

Kretschmer, T., Veenstra, R., Deković, M., \& Oldehinkel, A. J. (2017). Bullying development across adolescence, its antecedents, outcomes, and gender-specific patterns. Development and Psychopathology, 29(3), 941-955. https://doi. org/10.1017/S0954579416000596

Kwak, H., Kim, J., \& Kim, K. (2016). Examining the reciprocal causation between the experience of both school victimization and school bullying: Using autoregressive cross-lagged modeling. The Journal of Korea Elementary Education, 27(3), 37-56.

Kwon, J. (1999). Conceptualization of wangtta in Korea and methodological review of wangtta researches. Korean Journal of Psychology: Social Issues, 5(2), 59-72.

Lee, I. (2006). Collective ostracism among youth in Korea. In C. Daiute, Z. Beykont, C. Higson-Smith, \& L. Nucci (Eds.), International perspectives on youth conflict and development (pp. 124-138). New York, NY: Oxford University Press. 
Lee, J., Kwon, Y., Kim, E., Na, E., \& Park, S. (2016). An depth interview on Korean adolescent bullying: Focusing on bullying and social media. Journal of Communication Research, 53(1), 267-309.

Lee, S., Oh, I., \& Lee, J. (2014). Psychosocial characteristics of bullies, victims, bully-victims among elementary school students. Korean Journal of Youth Studies, 21(5), 391-416.

Lester, L., Cross, D., Shaw, T., \& Dooley, J. (2012). Adolescent bully-victims: Social health and the transition to secondary school. Cambridge Journal of Education, 42(2), 213-233. https://doi.org/10.1080/0305764X.2012.676630

Leventhal, T., \& Brooks-Gunn, J. (2000). The neighborhoods they live in: The effects of neighborhood residence on child and adolescent outcomes. Psychological Bulletin, 126(2), 309-337. https://doi.org/10.1037/0033-2909.126.2.309

Low, S., \& Espelage, D. (2014). Conduits from community violence exposure to peer aggression and victimization: Contributions of parental monitoring, impulsivity, and deviancy. Journal of Counseling Psychology, 61(2), 221-231.

Ministry of Education. (2013). Retireved from https://www.schoolinfo.go.kr/

Ministry of Education. (2018). Report of school violence in 2018. Sejong: School Life Culture Department.

Ministry of Health and Welfare. (2012). 2011 Health statistics of recipients of national basic living security program. Retrieved from http://www.mohw.go.kr/

Moon, B., Morash, M., \& McCluskey, J. D. (2012). General strain theory and school bullying: An empirical test in South Korea. Crime \& Delinquency, 58(6), 827-855. https://doi.org/10.1177/0011128710364809

Muthén, B., \& Muthén, L. K. (2000). Integrating person-centered and variable-centered analyses: Growth mixture modeling with latent trajectory classes. Alcoholism: Clinical and Experimental Research, 24(6), 882-891. https://doi.org/10.1111/j. 1530-0277.2000.tb02070.x

Muthén, L. K., \& Muthén, B. O. (2012). Mplus statistical modeling software: Release 7.0. Los Angeles, CA: Muthén \& Muthén.

Nansel, T. R., Craig, W., Overpeck, M. D., Saluja, G., \& Ruan, W. J. (2004). Cross-national consistency in the relationship between bullying behaviors and psychosocial adjustment. Archives of Pediatrics \& Adolescent Medicine, 158(8), $730-736$. https://doi.org/10.1001/archpedi.158.8.730

National Youth Policy Institute. (2017a). Codebook for KCYPS waves 1-7. Retrieved from https://www.nypi.re.kr/archive/ brdartcl/boardarticleList.do?brd_id=BDIDX_k9Fd9oFi29nooCcJ7kX1/4\&menu_nix=qi3a0s6n\&srch_ctgry_idx=CTIDX00042

National Youth Policy Institute. (2017b). User guide for KCYPS waves 1-7. Retrieved from https://www.nypi.re.kr/archive/ brdartcl/boardarticleList.do?brd_id=BDIDX_k9Fd9oFi29nooCcJ7kX114\&menu_nix=qi3a0s6n\&srch_ctgry_idx=CTIDX00042

Newman, D. A. (2003). Longitudinal modeling with randomly and systematically missing data: A simulation of ad hoc, maximum likelihood, and multiple imputation techniques. Organizational Research Methods, 6(3), 328-362. https://doi. org/10.1177/1094428103254673

No, U., Lee, H., Lee, E., \& Hong, S. (2017). Changes in student's school violence during middle school: Applying multivariate multilevel growth models to testing individual and school effects. Studies on Korean Youth, 28(1), 37-65.

Odgers, C. L., Caspi, A., Russell, M. A., Sampson, R. J., Arseneault, L., \& Moffitt, T. E. (2012). Supportive parenting mediates neighborhood socioeconomic disparities in children's antisocial behavior from ages 5 to 12 . Development and Psychopathology, 24(3), 705-721. https://doi.org/10.1017/S0954579412000326

Odgers, C. L., Moffitt, T. E., Tach, L. M., Sampson, R. J., Taylor, A., Matthews, C. L., \& Caspi, A. (2009). The protective effects of neighborhood collective efficacy on British children growing up in deprivation: A developmental analysis. Developmental Psychology, 45(4), 942-957. https://doi.org/10.1037/a0016162

Olweus, D. (2013). School bullying: Development and some important challenges. Annual Review of Clinical Psychology, 9, 751-780.

Park, J., \& Kim, H. (2015). A meta-analysis of factors having effects on school violence: Discovering the importance of School and Community factor. Korea Institute of Youth Facility \& Environment, 13(4), 115-128.

Park, Y., \& Oh, I. (2018). The relation between bullying experiences and bystanders behavioral reactions among elementary students - the moderating effects of peer conformity and perceived class norms. Asian Journal of Education, 19(1), 1-27.

Pellegrini, A. D., \& Bartini, M. (2000). A longitudinal study of bullying, victimization, and peer affiliation during the transition from primary school to middle school. American Educational Research Journal, 37(3), 699-725. https://doi.org/10.3102/ 00028312037003699

Pellegrini, A. D., \& Long, J. D. (2002). A longitudinal study of bullying, dominance, and victimization during the transition from primary school through secondary school. British Journal of Developmental Psychology, 20(2), 259-280. https://doi. org/10.1348/026151002166442

Reijntjes, A., Vermande, M., Goossens, F. A., Olthof, T., Van de Schoot, R., Aleva, L., \& Van der Meulen, M. (2013). Developmental trajectories of bullying and social dominance in youth. Child Abuse \& Neglect, 37(4), 224-234. https:// doi.org/10.1016/j.chiabu.2012.12.004

Ross, C. E. (2000). Neighborhood disadvantage and adult depression. Journal of Health and Social Behavior, 41(2), $177-187$. https://doi.org/10.2307/2676304

Ross, C. E., Reynolds, J. R., \& Geis, K. J. (2000). The contingent meaning of neighborhood stability for residents' psychological well-being. American Sociological Review, 65(4), 581-597. https://doi.org/10.2307/2657384 
Salmivalli, C., Lagerspetz, K., Björkqvist, K., Österman, K., \& Kaukiainen, A. (1996). Bullying as a group process: Participant roles and their relations to social status within the group. Aggressive Behavior, 22(1), 1-15. https://doi.org/10.1002/ (SICI)1098-2337(1996)22:1<1::AID-AB1>3.0.CO;2-T

Sampson, R. J., Raudenbush, S. W., \& Earls, F. (1997). Neighborhoods and violent crime: A multilevel study of collective efficacy. Science, 277(5328), 918-924. https://doi.org/10.1126/science.277.5328.918

Saunders, R., Buckman, J. E., Cape, J., Fearon, P., Leibowitz, J., \& Pilling, S. (2019). Trajectories of depression and anxiety symptom change during psychological therapy. Journal of Affective Disorders, 249, 327-335. https://doi.org/10.1016/j.jad.2019.02.043

Schmidt, C. J., Pierce, J., \& Stoddard, S. A. (2016). The mediating effect of future expectations on the relationship between neighborhood context and adolescent bullying perpetration. Journal of Community Psychology, 44(2), 232-248.

Scholte, R. H., Engels, R. C., Overbeek, G., De Kemp, R. A., \& Haselager, G. J. (2007). Stability in bullying and victimization and its association with social adjustment in childhood and adolescence. Journal of Abnormal Child Psychology, 35(2), 217-228. https://doi.org/10.1007/s10802-006-9074-3

Schwartz, S. H. (1990). Individualism-collectivism: Critique and proposed refinements. Journal of Cross-Cultural Psychology, 21(2), 139-157. https://doi.org/10.1177/0022022190212001

Seo, M. (2012). Subtypes of changes in overt and relational aggression and predictors of high-risk trajectories in children Journal of the Korean Society of Child Welfare, 38, 157-186.

Shaw, C. R., \& McKay, H. D. (1942). Juvenile delinquency and urban areas. Chicago, IL: University of Chicago Press.

Shaw, D. S., Gilliom, M., Ingoldsby, E. M., \& Nagin, D. S. (2003). Trajectories leading to school-age conduct problems. Developmental Psychology, 39(2), 189-200. https://doi.org/10.2307/1131832

Shin, J., Jang, G., \& Cho, Y. (2017). School violence prevention and understanding of students. Paju, South Korea: Yangseongwon.

Silver, E., Mulvey, E. P., \& Swanson, J. W. (2002). Neighborhood structural characteristics and mental disorder: Faris and Dunham revisited. Social Science \& Medicine, 55(8), 1457-1470. https://doi.org/10.1016/S0277-9536(01)00266-0

Singer, J. D., \& Willett, J. B. (2003). Applied longitudinal data analysis: Modeling change and event occurrence. New York, NY: Oxford University Press.

StataCorp. (2015). Stata Statistical Software: Release 14. College Station, TX: StataCorp LP.

Statistics Korea. (2018a). Retrieved from http://kosis.kr/index/index.do

Statistics Korea. (2018b). Social research in 2018. Seoul, South Korea: Social Statistics Bureau.

Tein, J. Y., Coxe, S., \& Cham, H. (2013). Statistical power to detect the correct number of classes in latent profile analysis. Structural Equation Modeling: A Multidisciplinary Journal, 20(4), 640-657. https://doi.org/10.1080/10705511.2013.824781

Teräsahjo, T., \& Salmivalli, C. (2003). "She is not actually bullied." The discourse of harassment in student groups. Aggressive Behavior, 29(2), 134-154. https://doi.org/10.1002/ab.10045

Triandis, H. C., Brislin, R., \& Hui, C. H. (1988). Cross-cultural training across the individualism-collectivism divide. International Journal of Intercultural Relations, 12(3), 269-289. https://doi.org/10.1016/0147-1767(88)90019-3

Ttofi, M. M., Farrington, D. P., \& Lösel, F. (2012). School bullying as a predictor of violence later in life: A systematic review and meta-analysis of prospective longitudinal studies. Aggression and Violent Behavior, 17(5), 405-418.

Volk, A. A., Dane, A. V., \& Marini, Z. A. (2014). What is bullying? A theoretical redefinition. Developmental Review, 34(4), 327-343.

Wang, J., lannotti, R. J., \& Nansel, T. R. (2009). School bullying among adolescents in the United States: Physical, verbal, relational, and cyber. Journal of Adolescent Health, 45(4), 368-375.

Wickrama, K., Mancini, J. A., Kwag, K., \& Kwon, J. (2012). Heterogeneity in multidimensional health trajectories of late old years and socioeconomic stratification: A latent trajectory class analysis. Journals of Gerontology Series B: Psychological Sciences and Social Sciences, 68(2), 290-297.

Xue, Y., Leventhal, T., Brooks-Gunn, J., \& Earls, F. J. (2005). Neighborhood residence and mental health problems of 5-to $11-$ year-olds. Archives of General Psychiatry, 62(5), 554-563.

Yeo, T., \& Kim, M. (2019). The effects of popularity and social achievement goal on aggression: Focusing on the gender differences. Journal of Education \& Culture, 25(1), 577-595.

How to cite this article: Han Y, Kim H, Ma J, Song J, Hong H. Neighborhood Predictors of Bullying Perpetration and Victimization Trajectories among South Korean Adolescents. J Community Psychol. 2019; 47:1714-1732. https://doi.org/10.1002/jcop.22226 